OPEN ACCESS

Edited by:

Dario De Medici,

Istituto Superiore di Sanità (ISS), Italy

Reviewed by:

Min Yue,

Zhejiang University, China

Alejandro Castillo,

Texas A\&M University, United States

*Correspondence:

Baowei Yang

ybw090925@163.com

${ }^{t}$ These authors have contributed equally to this work and share first authorship

Specialty section:

This article was submitted to

Food Microbiology,

a section of the journal

Frontiers in Microbiology

Received: 17 March 2020

Accepted: 08 June 2020

Published: 04 August 2020

Citation:

Li W, Li H, Zheng S, Wang Z,

Sheng $H$, Shi $C$, Shi $X$, Niu $Q$ and Yang B (2020) Prevalence, Serotype,

Antibiotic Susceptibility, and Genotype of Salmonella in Eggs From Poultry Farms and Marketplaces in Yangling, Shaanxi Province, China.

Front. Microbiol. 11:1482.

doi: 10.3389/fmicb.2020.01482

\section{Prevalence, Serotype, Antibiotic Susceptibility, and Genotype of Salmonella in Eggs From Poultry Farms and Marketplaces in Yangling, Shaanxi Province, China}

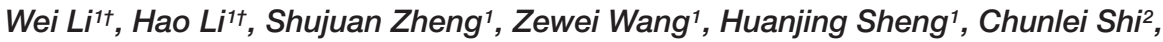 \\ Xianming Shi ${ }^{2}$ Qinya Niu ${ }^{1}$ and Baowei Yang ${ }^{1 *}$
}

${ }^{1}$ College of Food Science and Engineering, Northwest A\&F University, Yangling, China, ${ }^{2}$ MOST-USDA Joint Research Center for Food Safety, School of Agriculture and Biology, Shanghai Jiao Tong University, Shanghai, China

Poultry products such as eggs provide essential nutrients to the human body and thus play vital roles in the human food network. Salmonella is one of the most notorious foodborne pathogens and has been found to be prevalent in eggs. To better understand the characteristics of Salmonella in eggs, we investigated the prevalence of Salmonella spp. in 814 fresh eggs collected from poultry farms and retail marketplaces in Yangling, Shaanxi Province, China. The serotype, genotype, and antibiotic susceptibilities of 61 Salmonella isolates recovered from the eggs were analyzed. The average detection rate of Salmonella-positive eggs was $5.6 \%$, with $6.6 \%$ of the eggs collected from poultry farms and $5.1 \%$ from marketplaces. Thirteen serotypes were identified from the 61 isolates, among which Salmonella Typhimurium (24.5\%) and Salmonella Indiana (22.9\%) were the most prevalent serotypes. Other dominant serotypes included Salmonella Thompson (13.1\%) and Salmonella Enteritidis (11.4\%), with the remaining nine serotypes detected at low rates (1.6-4.9\%). All the Salmonella isolates tested were resistant to sulfisoxazole (100.0\%). The majority $(77.1 \%)$ of the isolates were resistant to nalidixic acid, amoxicillin-clavulanate, and ampicillin, while nearly two-thirds (63.968.9\%) were resistant to trimethoprim-sulfamethoxazole, kanamycin, tetracyclines, and chloramphenicol. The rate of resistance to ciprofloxacin was $40.1 \%$; the resistance rates to streptomycin, ceftiofur, and ceftriaxone ranged from 21.3 to $26.2 \%$; and those to gentamicin, amikacin, and cefoxitin were relatively low (3.3-16.4\%). Forty-nine (80.3\%) Salmonella isolates exhibited resistance to multiple antibiotics, 20 (32.8\%) of which were resistant to at least 10 antibiotics. Subtyping by pulse-field gel electrophoresis revealed a close genetic relatedness of Salmonella isolates from poultry farms, in striking contrast to the high diversity of the isolates obtained from marketplaces. Isolates of the same serotype always shared identical genotype and antibiotic resistance profiles, even the ones that were recovered from eggs sampled at different locations and times. These findings indicate that diverse Salmonella spp. with high rates of multidrug resistance are prevalent in fresh eggs in the study area. More attention should be paid to egg production, transportation, and storage to prevent foodborne outbreaks caused by Salmonella.

Keywords: antibiotic resistance, eggs, genotype, Salmonella, serotype 


\section{INTRODUCTION}

Salmonella spp. are notorious foodborne pathogens that can cause diarrhea in humans and animals (Cowen et al., 2016). Reportedly, there are approximately 9.38 million cases of Salmonella infections and 15,000 deaths from the infection worldwide every year (Patchanee et al., 2017). Among the various vehicles of Salmonella, poultry and poultry products are not only identified as the predominant reservoirs, but are also considered to be the primary sources of human salmonellosis based on evidence from epidemiological traceback investigations (Andino and Hanning, 2015; McWhorter and Chousalkar, 2019). A previous study has indicated that the egg white possesses unique physical and biochemical properties, creating a complex antimicrobial environment to resist antigens (Huang et al., 2019). However, egg white and egg yolk membranes are the major infection sites for Salmonella, which means that Salmonella still has the opportunity to survive in the resistant environment of eggs (Raspoet et al., 2019).

Currently, the outbreak of foodborne diseases caused by the consumption of eggs contaminated with Salmonella remains severe worldwide. According to reports from the United States Food and Drug Administration (FDA) and the Centers for Disease Control and Prevention (CDC), there were 52 foodborne outbreaks in Missouri in 2015 due to eggs contaminated by Salmonella Oranienburg. In 2016, the CDC again notified the FDA of eight clinical cases from three states, i.e., Illinois, Kansas, and Missouri, which were closely related to the hereditary strains of the S. Oranienburg outbreak in 2015 (FDA, 2019c). In March 2018, the FDA learned about 45 Salmonella Braenderup-infected consumers in 10 states, 11 of whom were hospitalized without death. The outbreak was tracked down to Rose Acre Farms' Hyde County farm in North Carolina and 207 million eggs were recalled around the United States (FDA, 2019a).

Recently, Salmonella was detected in eggs collected from 41 (63.5\%) of 63 farms in Australia that underwent environmental sampling (Moffatt et al., 2017). In addition, 88.6\% (124/140) of the eggs and all of the 19 farms sampled in the western region of Cameroon were found to be Salmonella-positive (Kouam et al., 2018). So far, multiple Salmonella serotypes including Salmonella Typhimurium, Salmonella Indiana, and Salmonella Enteritidis have been commonly detected from eggs, poultry, and poultry farm environments (Moffatt et al., 2017). To prevent infection by pathogenic bacteria and promote the growth of laying hens, a large number of antibiotics are used in the feeding process. A nationwide market survey indicated that the total amount of 36 antibiotics that are frequently detected in livestock farms, wastewater treatment plants, and environment settings reached 92,700 tons in China in 2015. Notably, the rate of antibiotic usage in poultry farms was as high as $19.6 \%(18,100 / 92,700)$ (Zhang et al., 2015).

$\beta$-Lactams, cephalosporins, and fluoroquinolones are the most frequently used antibiotics in the poultry industry (Fardsanei et al., 2017). Taking into account the antibiotics used in other fields, Salmonella has developed high resistance to a broad range of antibiotics, leading to increased healthcare costs and clinical treatment failure (Cui et al., 2016). Many studies have characterized Salmonella in poultry and poultry-associated foods globally, especially eggs (Moffatt et al., 2017; Kouam et al., 2018; Li et al., 2018; FDA, 2019a,b,c; Karimiazar et al., 2019; Kingsbury et al., 2019; Sanchez-Salazar et al., 2019). Evidence suggests that the genetic diversity of Salmonella isolated from various sources is abundant, whereas the isolates from the same poultry farms are closely related (Xie et al., 2019). Moreover, the occurrence and characteristics of Salmonella in poultry products including eggs always show dynamic variation during production, storage, and handling (Li et al., 2018). Therefore, it is of great significance to continuously monitor this group of pathogens in eggs to ensure food safety.

In this study, we characterized Salmonella isolates from fresh eggs in poultry farms and retail marketplaces in Yangling, Shaanxi Province, China. The objective of the study was to further explore the prevalence and transmission of Salmonella during egg production and sales links in order to prevent Salmonella outbreaks.

\section{MATERIALS AND METHODS}

\section{Sample Collection}

A total of 814 fresh eggs were collected in Yangling and its surrounding districts in Shaanxi Province, China, from mid2013 to late 2014. Specifically, 304 eggs were collected from three different poultry farms. Farm $\mathrm{X}$ in Xiajiagou Village is a large modern laying hen farm with fully automated cage raising equipment, including equipment for heating, ventilation, water supply, feeding, egg collection, manure removal, cages, and lighting. In this farm, there are approximately 20,000 laying hens with a breeding density of $9-10 / \mathrm{m}^{2}$. Farm C in Cuixigou Village is a semi-automated laying hen farm of a medium production scale, with basic equipment for heating, ventilation, cages, lighting, and water feeding. In this farm, there are approximately 9000 laying hens with a breeding density of $11-12 / \mathrm{m}^{2}$. Farm F in Fuzeyuan Company is the smallest laying hen farm with basic equipment for heating, ventilation, cages, and lighting. In this farm, there are approximately 4000 laying hens with a breeding density of $9-10 / \mathrm{m}^{2}$. Additionally, 510 eggs of seven brands were collected from seven supermarkets (eight retail outlets) and four wet markets (10 retail outlets).

At each sampling location, the eggs were sampled weekly in August, September, and October 2013 and in March, June, August, October, and November 2014. At each sampling time, three, six, or nine eggs (depending on the total number of eggs for sale) were collected at random in a supermarket or wet market. In each poultry farm, no more than 50 eggs were collected at random from egg-laying troughs. The egg samples were delivered to the Microbiology Laboratory in the College of Food Science and Engineering, Northwest A\&F University (Yangling, Shaanxi Province China) for Salmonella isolation within $12 \mathrm{~h}$ of collection.

\section{Isolation and Identification of Salmonella}

Salmonella isolates were recovered from egg samples according to the method described by Yang et al. (2010) with some 
TABLE 1 | Antibiotic concentration ranges and data interpretation for the susceptibility test of Salmonella isolates.

\begin{tabular}{|c|c|c|c|c|c|}
\hline Antibiotic agent & Abbreviation & Antibiotic concentration range $(\mu \mathrm{g} / \mathrm{mL})$ & \multicolumn{3}{|c|}{ Breakpoint interpretive criteria $(\mu \mathrm{g} / \mathrm{mL})^{*}$} \\
\hline \multicolumn{6}{|l|}{ Aminoglycosides } \\
\hline Amikacin & AMK & $0.5-64$ & $\leq 16$ & 32 & $\geq 64$ \\
\hline Gentamicin & GEN & $0.25-16$ & $\leq 4$ & 8 & $\geq 16$ \\
\hline Kanamycin & KAN & $1-64$ & $\leq 16$ & 32 & $\geq 64$ \\
\hline Amoxicillin-clavulanic acid & AMC & $0.25 / 0.12-32 / 16$ & $\leq 8 / 4$ & $16 / 8$ & $\geq 32 / 16$ \\
\hline Ampicillin & AMP & $0.5-32$ & $\leq 8$ & 16 & $\geq 32$ \\
\hline \multicolumn{6}{|c|}{ Cephalosporins (3rd and 4th generations) } \\
\hline Cefoxitin & FOX & $2-32$ & $\leq 8$ & 16 & $\geq 32$ \\
\hline Ceftriaxone & $\mathrm{CRO}$ & $0.03-4$ & $\leq 8$ & $16-32$ & $\geq 64$ \\
\hline Ceftiofur & $\mathrm{TIO}$ & $0.25-8$ & $\leq 8$ & $16-32$ & $\geq 64$ \\
\hline Ciprofloxacin & CIP & $0.004-4$ & $\leq 0.06$ & $0.12-0.5$ & $1 \geq$ \\
\hline \multicolumn{6}{|l|}{ Tetracyclines } \\
\hline Tetracyclines & TET & $0.5-16$ & $\leq 4$ & 8 & $\geq 16$ \\
\hline \multicolumn{6}{|l|}{ Chloramphenicol } \\
\hline Chloramphenicol & $\mathrm{CHL}$ & $2-32$ & $\leq 8$ & 16 & $\geq 32$ \\
\hline \multicolumn{6}{|l|}{ Folate pathway inhibitors } \\
\hline Sulfisoxazole & FIS & 8-512 & $\leq 256$ & NA & $\geq 512$ \\
\hline Trimethoprim-sulfamethoxazole & SXT & $0.5 / 9.5-4 / 76$ & $\leq 2 / 38$ & NA & $\geq 4 / 76$ \\
\hline
\end{tabular}

${ }^{*}$ S, sensitive; I, intermediate resistance; and R, resistance. "No CLSI interpretative criteria are available for streptomycin; thus, provisional breakpoint from NARMS was used.

modifications. Briefly, each egg (ca. $60 \mathrm{~g}$, including the shell) was broken and uniformly mixed with $540 \mathrm{~mL}$ of buffered peptone water (BD, Cockeysville, MD, United States) in a homogenization bag, then homogenized for $2 \mathrm{~min}$. Before handling the next egg, a new pair of germ-free gloves was used to avoid bacterial cross-contamination throughout the process. The mixed cultures were pre-incubated at $37^{\circ} \mathrm{C}$ with shaking at $100 \mathrm{rpm}$ for $6 \mathrm{~h}$. Subsequently, $10 \mathrm{~mL}$ cultures were transferred into $100 \mathrm{~mL}$ of tetrathionate broth (TT; BD) and $1 \mathrm{~mL}$ cultures were transferred to $100 \mathrm{~mL}$ of Rappaport-Vassiliadis broth (RV; BD). The inoculated TT and RV broths were incubated at $42^{\circ} \mathrm{C}$ with shaking at $100 \mathrm{rpm}$ for $24 \mathrm{~h}$. Afterward, TT cultures were streaked on to xylose lysine tergitol 4 agar (XLT4; BD) and RV cultures were streaked on to xylose lysine deoxycholate agar (XLD; BD). The inoculated XLT4 and XLD plates were incubated at $35^{\circ} \mathrm{C}$ for $48 \mathrm{~h}$, and two putative colonies with a typical Salmonella phenotype were picked from each plate and subcultured on a fresh XLT4 plate for purification. The purified isolates were stabbed into one triple sugar iron (BD) slant and one ureaagar (BD) slant, respectively, followed by incubation at $35^{\circ} \mathrm{C}$ for 18-24 h to exclude Escherichia coli and Proteus species.

Presumptive Salmonella isolates were identified by polymerase chain reaction (PCR) using the primers invAF (5'-GTGAAATTATCGCCACGTTCGGGCAA- $3^{\prime}$ ) and invAR (5'-TCATCGCACCGTCAAAGGAACC-3') (Rahn et al., 1992). Polymerase chain reaction was carried out in a $25-\mu \mathrm{L}$ reaction mixture containing $0.5 \mu \mathrm{M}$ of each primer, $250 \mu \mathrm{M}$ of dNTP,
$1 \times$ PCR buffer, $1.5 \mathrm{mM} \mathrm{MgCl}_{2}, 0.5 \mathrm{U}$ of Taq DNA polymerase (TaKaRa, Dalian, China), and $5 \mu \mathrm{L}$ of DNA template. All PCR reactions were performed on a MyCircle thermocycler (Bio-Rad, Hercules, CA, United States) with pre-denaturation at $95^{\circ} \mathrm{C}$ for $5 \mathrm{~min}$, followed by 35 cycles of $95^{\circ} \mathrm{C}$ for $30 \mathrm{~s}, 64^{\circ} \mathrm{C}$ for $30 \mathrm{~s}$, and $72^{\circ} \mathrm{C}$ for $30 \mathrm{~s}$, and a final extension of $72^{\circ} \mathrm{C}$ for $5 \mathrm{~min}$. Polymerase chain reaction products were stained with ethidium bromide and visualized under UV light after gel electrophoresis in 1\% agarose (Kasturi and Drgon, 2017). S. Typhimurium LT2 was used as the positive control strain.

\section{Serotyping of Somatic and Flagellar Antigens}

After PCR identification, Salmonella isolates were serotyped in the Henan Center of Disease Control and Prevention (Zhengzhou, Henan Province, China). Somatic (O) and flagellar $(\mathrm{H})$ antigens were determined by the slide agglutination method using Salmonella-specific hyperimmune sera (S\&A Company, Bangkok, Thailand). The serotype was identified by the antigen form according to the Kauffman-White scheme (Ben Aissa et al., 2007). S. Typhimurium LT2 was used as the positive control strain.

\section{Antibiotic Susceptibility Test}

Table 1 lists the antibiotics used for the susceptibility test. The agar dilution method suggested by the Clinical and Laboratory 
Standards Institute (CLSI, 2015) was used to determine the minimum inhibitory concentrations of the antibiotics to Salmonella isolates on Mueller-Hinton agar (Beijing Land Bridge Technology Co., Ltd, Beijing, China). The results of resistance, intermediate resistance, and susceptibility were interpreted via the guidelines established by the CLSI, except for streptomycin, the breakpoint of which was interpreted by that specified by the National Antimicrobial Resistance Monitoring System (U.S. Food and Drug Administration, 2013; FAO, 2018a,b). E. coli ATCC25922 and ATCC35218 and Enterococcus faecalis ATCC29212 were used as the quality control strains.

\section{Subtyping by Pulse-Field Gel Electrophoresis}

Pulse-field gel electrophoresis (PFGE) was used to determine the genetic relationship between Salmonella isolates according to the PulseNet protocol (Centers for Disease Control and Prevention [CDC], 2013). Briefly, Salmonella subcultures were grown on Luria-Bertani agar overnight at $37^{\circ} \mathrm{C}$ to reach an optical density of 0.5 and then embedded in SeaKem Gold agarose (Lonza, Rockland, ME, United States). Then culture plugs were lysed in cell lysis buffer with $100 \mu \mathrm{g} \mathrm{mL}^{-1}$ protease $\mathrm{K}$ (TaKaRa) by incubation at $55^{\circ} \mathrm{C}$ in a shaking water bath for $2 \mathrm{~h}$. Subsequently, the lysed plugs were washed twice with sterile water and then washed four times with sterilized Tris-EDTA buffer. A slice was cut from the plug and digested using $50 \mathrm{U}$ of $\mathrm{XbaI}$ (TaKaRa) by incubation in a $37^{\circ} \mathrm{C}$ water bath for $1.5-2 \mathrm{~h}$. The digested DNA fragments in each slice were separated in $0.5 \times$ Tris-borate-EDTA buffer at $14^{\circ} \mathrm{C}$ for $20 \mathrm{~h}$ using a Chef Mapper electrophoresis system (Bio-Rad) with pulse times of 2.16-63.8 s. The gel was stained using ethidium bromide $\left(100 \mu \mathrm{g} \mathrm{mL}^{-1}\right)$, and the DNA bands were visualized via UV transillumination (Bio-Rad). Pulsefield gel electrophoresis results were manually analyzed using BioNumerics v3.0 (Applied Maths, Kortrijk, Belgium), and the extent of variability was determined using the Dice coefficient and clustering by the unweighted pair-group average method (Yang et al., 2014). S. Braenderup H9812 was used as the standard DNA control.

\section{Statistical Analysis}

Statistical analysis was performed using IBM SPSS Statistics v22 (IBM Corp., Armonk, NY, United States). Pearson's chi-square test was used to determine the differences in the detection rates of Salmonella-positive samples and Salmonella serotypes in eggs across different sampling locations (i.e., wet markets, supermarkets, and poultry farms) and times. The results were analyzed at the 5\% ( $\alpha=0.05)$ level to determine whether differences were significant. Data were edited using Microsoft Excel 2016 (Microsoft Corp., Redmond, WA, United States), with environmental variables (i.e., serotype, sampling location, and sampling time) and explanatory environmental variables (i.e., number and category of the antibiotics to which Salmonella exhibited resistance) organized into the form of "row representing samples and column representing variables." The edited data were imported into Canoco v5.0 (Microcomputer Power, Ithaca, NY, United States) for redundancy analysis. The
TABLE 2 | Prevalence of Salmonella in eggs collected from poultry farms and marketplaces at different sampling locations and times.

\begin{tabular}{|c|c|c|c|}
\hline & $\begin{array}{l}\text { Location or time } \\
\text { (egg number) }\end{array}$ & $\begin{array}{c}\text { Number (percentage) } \\
\text { of Salmonella-positive } \\
\text { eggs }\end{array}$ & $\begin{array}{c}\text { Number (percentage) } \\
\text { of Salmonella } \\
\text { isolates }\end{array}$ \\
\hline \multirow[t]{2}{*}{ Source } & Retail market (510) & $26(5.1)$ & $40(65.6)$ \\
\hline & Poultry farm (304) & $20(6.6)$ & $21(34.4)$ \\
\hline Retail & Supermarket (259) & $15(5.8)$ & $22(36.1)$ \\
\hline market & Wet market (251) & $11(4.4)$ & $18(29.5)$ \\
\hline Poultry & C $(88)$ & $19(21.6)^{\# \#}$ & $20(32.8)$ \\
\hline \multirow[t]{2}{*}{ farm } & $X(108)$ & $1(0.9)$ & $1(1.6)$ \\
\hline & $F(108)$ & 0 & 0 \\
\hline Sampling & $2013(134)$ & $2(1.5)$ & $2(3.3)$ \\
\hline year & 2014 (680) & $44(6.5)^{\star}$ & $59(96.7)$ \\
\hline Sampling & Aug. 2013 (98) & $2(2.0)^{\mathrm{c}}$ & 2 \\
\hline \multirow[t]{7}{*}{ month } & Spt. 2013 (12) & $0^{C}$ & 0 \\
\hline & Oct. 2013 (24) & $0^{\mathrm{C}}$ & 0 \\
\hline & Mar. 2014 (66) & $20(30.3)^{a}$ & 33 \\
\hline & Jun. 2014 (175) & $4(2.3)^{\mathrm{C}}$ & 5 \\
\hline & Aug. 2014 (135) & $0^{c}$ & 0 \\
\hline & Oct. 2014 (196) & $19(9.7)^{\mathrm{b}}$ & 20 \\
\hline & Nov. 2014 (108) & $1(0.9)^{\mathrm{c}}$ & 1 \\
\hline Total & 814 & $46(5.6)$ & $61(100)$ \\
\hline
\end{tabular}

${ }^{\# \#} P<0.01 ;{ }^{*} P<0.05, P=0.023$. ${ }^{a, b, c}$ Values (percentage) with different superscripted letters are significantly different between groups.

rate of antibiotic-resistant Salmonella isolates between different serotypes was analyzed using $\mathrm{R} v 3.4 .4^{1}$, and a heatmap was created using the R package "pheatmap" v3.0.12.

\section{RESULTS}

\section{Prevalence of Salmonella}

Of the 814 eggs, 46 (5.6\%) were detected to be Salmonellapositive (Table 2). The detection rate of Salmonella-positive eggs from poultry farms was slightly higher $(6.6 \%, 20 / 304)$ than that from retail markets $(5.1 \%, 26 / 510)$. Among the different marketplaces, the detection rate of Salmonella-positive eggs was slightly higher in supermarkets $(5.8 \%, 15 / 259)$ than in wet markets $(4.4 \%, 11 / 251)$. However, the differences in the detection rates of Salmonella-positive eggs were not significant among retail markets and poultry farms.

Among the three poultry farms, no Salmonella-positive eggs were detected from farm F, only one $(0.9 \%)$ Salmonella-positive egg was detected in farm X, while 19 (21.6\%) were detected in farm C. The detection rate of Salmonella in eggs from farm $\mathrm{C}$ was significantly $(P<0.01)$ higher than those from the other two poultry farms. During the investigation period, the detection rate of Salmonella-positive eggs was significantly higher $(P<0.05)$ in $2014(6.5 \%, 44 / 680)$ than in $2013(1.5 \%, 2 / 134)$. Additionally, the detection rates of Salmonella-positive eggs in March 2014 (30.3\%, 20/60) and October 2014 (9.7\%, 19/196)

\footnotetext{
${ }^{1}$ https://rstudio.com/

${ }^{2}$ https://www.rdocumentation.org/packages/pheatmap/versions/1.0.12
} 


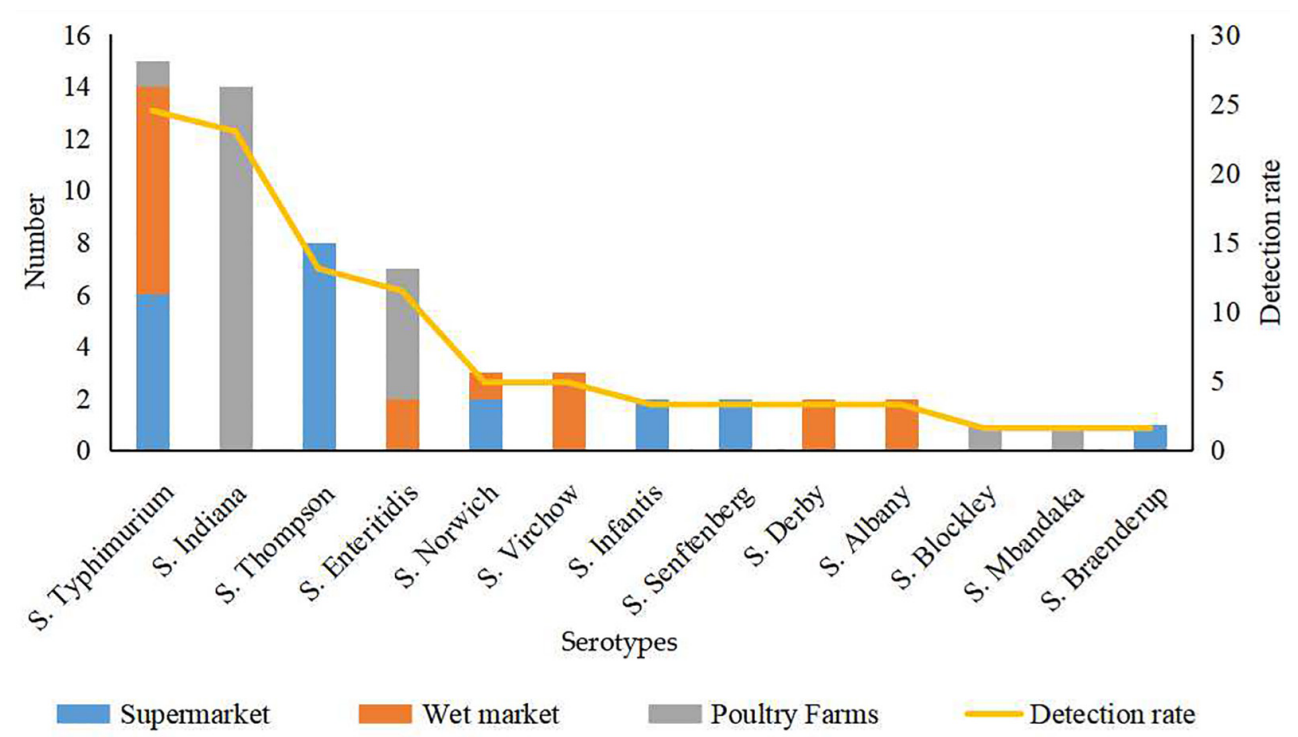

FIGURE 1 | Serotype of 61 Salmonella isolates from eggs from different sources.

were significantly $(P<0.05)$ higher than those from the other sampling times (Table 2).

In total, 61 Salmonella isolates were recovered from XLD and XLT4 plates derived from the 46 Salmonella-positive eggs. Among them, 40 (65.6\%) isolates were obtained from eggs collected from retail markets and 21 (34.4\%) were from eggs sampled from poultry farms (Table 2).

\section{Serotype of Salmonella}

Thirteen serotypes were identified from the 61 Salmonella isolates, among which $S$. Typhimurium (24.5\%) and $S$. Indiana $(22.9 \%)$ were the most commonly detected serotypes (Figure 1). The detection rates of Salmonella Thompson (13.1\%) and Salmonella Enteritidis (11.4\%) were moderately high. Additionally, Salmonella Norwich and Salmonella Virchow accounted for $4.9 \%$ of all the identified isolates each; Salmonella Derby, Salmonella Senftenberg, Salmonella Infantis, and Salmonella Albany accounted for 3.2\% each; and Salmonella Blockley, Salmonella Mbandaka, and $S$. Braenderup were detected at the lowest rates (1.6\% each).

The 39 Salmonella isolates recovered from retail eggs covered 10 serotypes, while the 22 isolates recovered from poultry farms covered five serotypes (Figure 1). Considering their source, all $S$. Indiana, $S$. Blockley, and $S$. Mbandaka isolates and the majority (71.4\%) of $S$. Enteritidis isolates were from poultry farms. All $S$. Thompson, S. Infantis, S. Senftenberg, and S. Braenderup isolates and $40.0 \%$ of $S$. Typhimurium isolates were from supermarkets. All S. Virchow, S. Derby, and $S$. Albany isolates and more than half of S. Norwich (66.7.\%) and S. Typhimurium (53.3\%) isolates were from wet markets.

\section{Antibiotic Susceptibility of Salmonella}

All the 61 Salmonella isolates were resistant to sulfisoxazole (100\%), and the rate of sulfisoxazole resistance was significantly
$(P<0.05)$ higher than the rates of resistance to the other 13 antibiotics tested for (Figure 2). For example, $77.1 \%$ of the isolates were resistant to amoxicillin-clavulanate, ampicillin, and nalidixic acid, while nearly two-thirds (63.9-68.9\%) of the tested isolates exhibited resistance to trimethoprim-sulfamethoxazole, kanamycin, tetracyclines, and chloramphenicol. Less than half $(40.1 \%)$ of the isolates were resistant to ciprofloxacin; around one-fifth (21.3-26.2\%) of the isolates were resistant to streptomycin, ceftiofur, and ceftriaxone; and the resistance rates to gentamicin, amikacin, and cefoxitin were relatively low (3.3$16.4 \%)$.

There were no significant differences in the resistance rates of Salmonella isolates to amoxicillin-clavulanate, ampicillin, and nalidixic acid; however, these resistance rates were significantly $(P<0.05)$ higher than the rates of resistance to the other antibiotics tested, except the resistance rate to sulfisoxazole. Additionally, significant $(P<0.05)$ differences were found in the resistance rates to amikacin, gentamicin, kanamycin, streptomycin, chloramphenicol, cefoxitin, ceftiofur, ceftriaxone, ciprofloxacin, and trimethoprim-sulfamethoxazole. Moreover, the resistance rates to aminoglycosides (amikacin, gentamicin, and kanamycin) and cephalosporins (cefoxitin, ceftriaxone, and ceftiofur) differed significantly $(P<0.05$; Figure 2$)$.

A total of $49(80.3 \%)$ Salmonella isolates were resistant to more than three categories of antibiotics. Specifically, 10 (20.4\%) isolates were resistant to 3-5 categories (3-8 species) of antibiotics and the remaining $39(79.6 \%)$ isolates were resistant to $6-8$ categories ( $8-12$ species) of antibiotics. The Salmonella isolates that were resistant to the 15 antibiotics based on different serotypes could be grouped into two clusters (G1 and G2; Figure 3).

Isolates resistant to ceftriaxone, amikacin, ciprofloxacin, ceftiofur, nalidixic acid, kanamycin, chloramphenicol, and cefoxitin were grouped in cluster G1. Of these, isolates resistant 


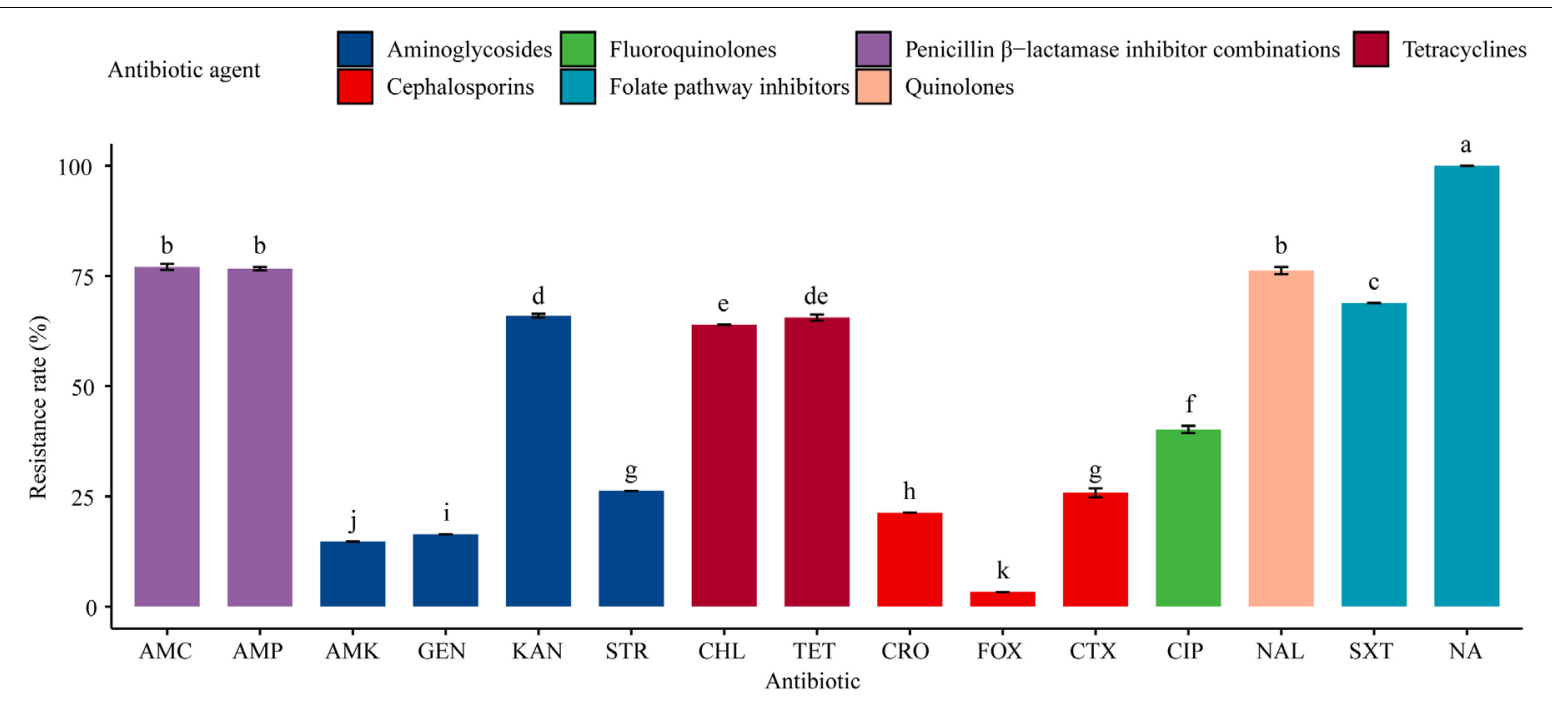

FIGURE 2 | Resistance of 61 Salmonella isolates to 15 antibiotics. Table 1 has a list of abbreviations used in the abscissa. Different colors represent seven different antibiotic categories. Data are mean \pm standard deviation $(n=3)$. Different letters used to label the bar chart denote significant differences found between the resistance rates of isolates to the corresponding antibiotic $(P<0.05)$.

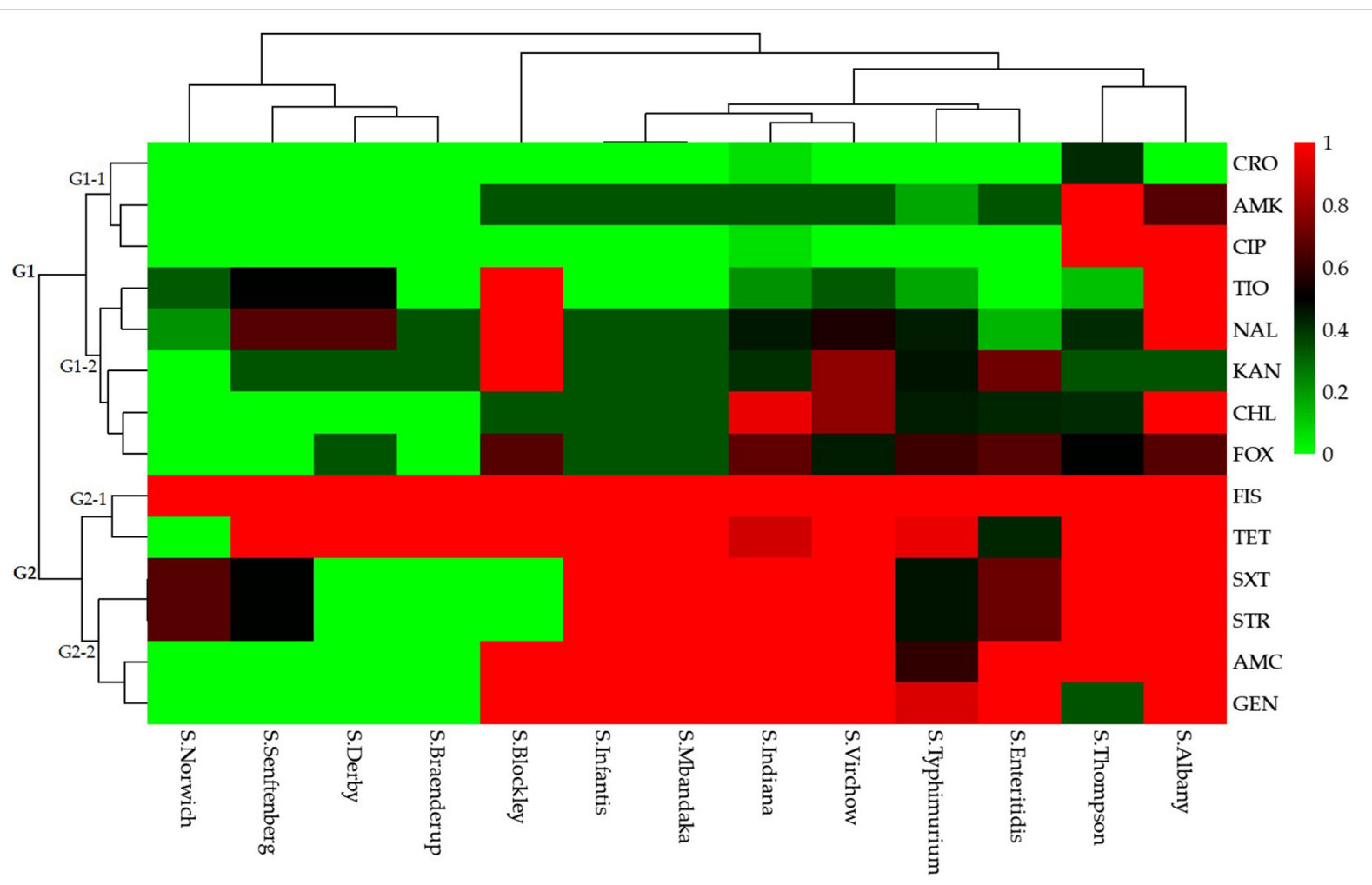

FIGURE 3 | Heatmap (with dendrograms) showing the antibiotic susceptibilities of 61 Salmonella isolates among 13 serotypes. Red squares (pixels) represent high-frequency values and green squares represent low-frequency values. Table 1 presents a list of the abbreviations of the antibiotics.

to ceftriaxone, amikacin, and ciprofloxacin were grouped in a secondary cluster (G1-1), and isolates resistant to the other five antibiotics were grouped in another secondary cluster (G1-2). Isolates resistant to sulfisoxazole, tetracyclines, trimethoprimsulfamethoxazole, streptomycin, amoxicillin-clavulanate, and gentamicin were grouped in cluster G2. In this cluster, isolates resistant to sulfisoxazole and tetracycline were grouped in a secondary cluster (G2-1), and those resistant to trimethoprimsulfamethoxazole, streptomycin, amoxicillin-clavulanate, and gentamicin were grouped in another secondary cluster (G2-2). 


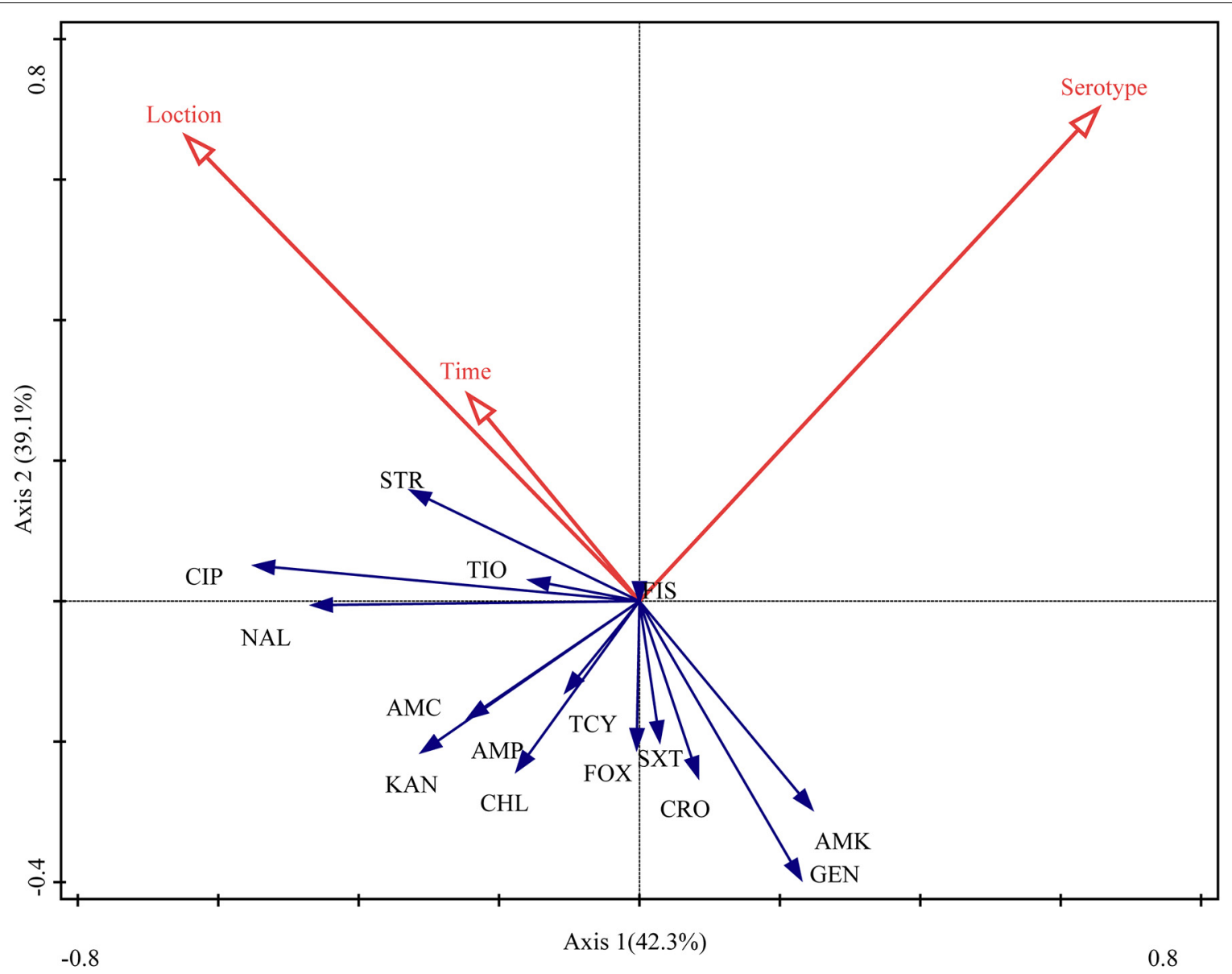

FIGURE 4 | Relationship between the antibiotic susceptibility of Salmonella isolates, their serotype, sampling location, and sampling time. Table 1 presents a list of the abbreviations of the antibiotics. Blue arrow, species variable; red arrow, environmental variable. The cosine of the angle between two variables represents the correlation between them. For example, two variables that are nearly at a right angle to each other are almost uncorrelated.

The RDA biplot (Figure 4) indicated that the contribution of serotype to the antibiotic susceptibility of Salmonella isolates was the highest $(42.3 \%, P=0.004)$, followed by sampling location $(39.1 \%, P=0.018)$ and time $(18.6 \%, P=0.138)$. The serotype of the isolates was correlated with their susceptibility to most of the antibiotics tested for, except amoxicillin-clavulanate, ampicillin, kanamycin, and chloramphenicol. Additionally, sampling location and time were (closely) correlated with the susceptibility of the isolates to streptomycin, ciprofloxacin, ceftiofur, nalidixic acid, amoxicillin-clavulanate, ampicillin, kanamycin, tetracyclines, and chloramphenicol.

\section{PFGE Subtype of Salmonella}

When the Salmonella isolates were subtyped by PFGE, the genomic DNA of each isolate produced 13-21 bands with the typing rate of $100 \%$ (Figure 5). Isolates derived from the eggs collected in the same month always belonged to the same serotype, and they exhibited the same or similar antibiotic resistance phenotype, despite being recovered from different locations (C1-1, C2-3, and C5). In contrast, some of the isolates recovered from the eggs sampled from different locations belonged to various serotypes, yet they also showed identical PFGE profiles and antibiotic resistance phenotypes (C1-2 and C1-3). Moreover, some isolates recovered from the eggs collected at the same location and time belonged to the same serotype, but they showed distinct PFGE and antibiotic resistance profiles (C2-2, C2-4, and C2-5).

\section{DISCUSSION}

The total egg production reached 24,446 million metric tons in 2013 and 4539 billion in 2014 (FAO, 2018a,b). If contaminated eggs were produced by laying hens infected with Salmonella, it would be difficult to effectively eliminate such contamination through vaccination and other interventions. Therefore, not only could this lead to outbreaks of foodborne illness in humans, but chickens hatched from contaminated eggs may also have inherent defects (Barrow, 2007; Kouam et al., 2018). The detection and characterization of Salmonella spp. in eggs can provide useful information for the mitigation of socioeconomic losses caused by contamination with Salmonella. In the present 


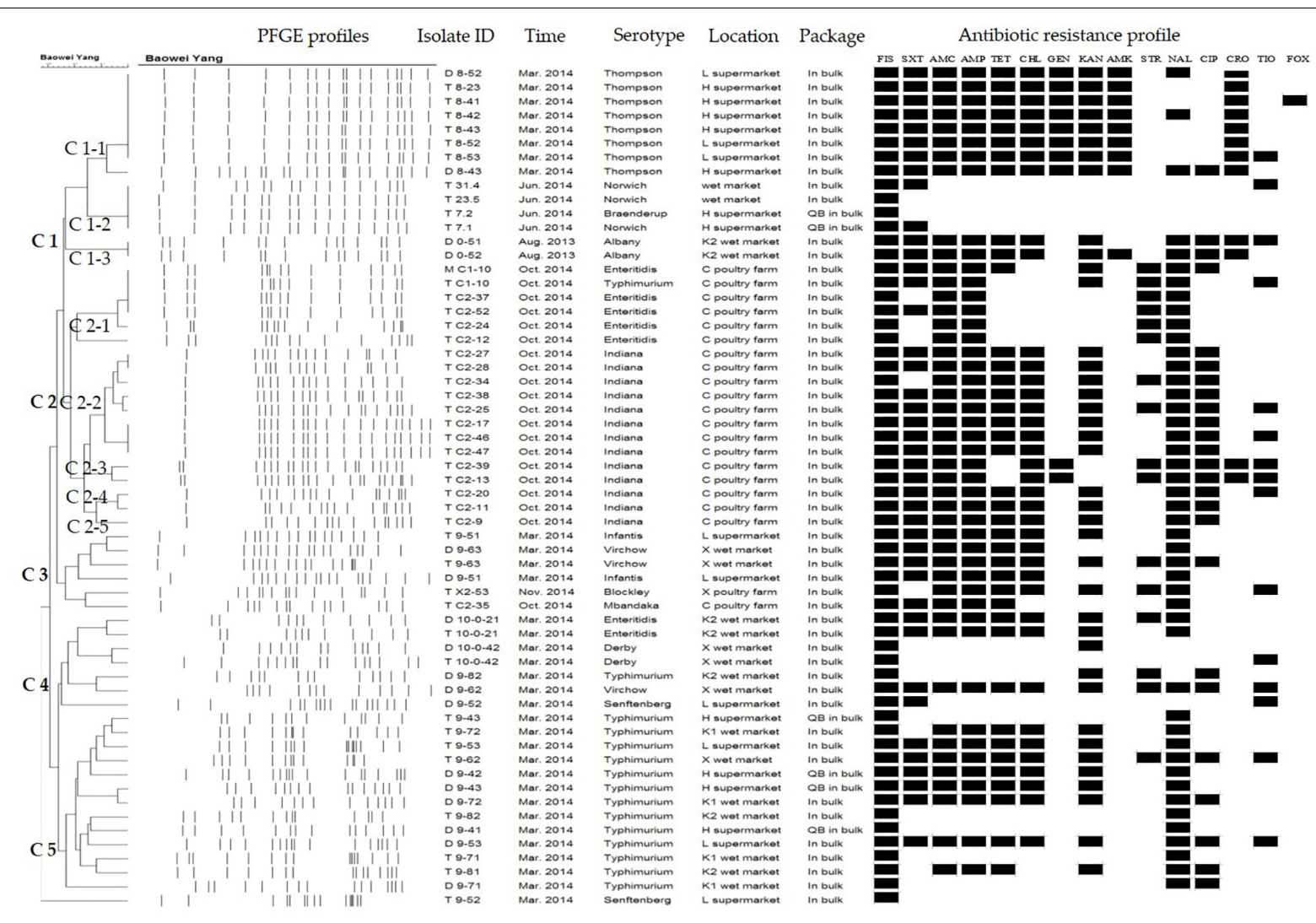

FIGURE 5 | Xbal-based pulse-field gel electrophoresis (PFGE) and antibiotic resistance profiles of Salmonella isolates from eggs.

study, the average detection rate of Salmonella-positive eggs in poultry farms, supermarkets, and wet markets in Yangling (5.6\%) was higher than those reported in Shandong Province (2.1\%, 49/2342) and Hebei Province (4.8\%) (Li et al., 2018; Yang et al., 2019); however, it was much lower than the detection rates in some conventional farms in Sichuan Province (12.2\%) and Jiangsu Province (17.4\%, 160/920), China (Li et al., 2013; Hai et al., 2020). During the study period, a high detection rate of Salmonella-positive eggs in 2014 was mainly found in the samples collected in March and October, and all the Salmonella-positive samples in October were obtained from the medium-scale poultry farm C. Although these results indicate that Salmonella is still prevalent in the eggs, the tendency of reduced prevalence suggests that in recent years, China has achieved remarkable results in monitoring and controlling the contamination of eggs with pathogens including Salmonella from farm to table.

Based on our results, the detection rate of Salmonella-positive eggs in the production (6.6\%) and sales (5.1\%) links were similar. A previous investigation provided a detailed description of contamination during five breeder farm production stages (i.e., laying, hatching, rearing, brooding, and post-hatching), with a considerably high prevalence of egg samples containing Salmonella found at the laying (29.2\%) and hatching $(21.6 \%)$ stages (Fei et al., 2017). In the current study, a total of 304 eggs were collected from three different poultry farms of different scales in Yangling. Although the number of eggs collected from each farm was similar, the detection rate of Salmonella-positive eggs varied in a broad range across different farms. Except one from the large-scale farm X, all the remaining Salmonellapositive eggs were detected from medium-scale farm C, while no Salmonella-positive eggs were detected from the smallscale farm F. Farm C was a conventional poultry farm with a smaller production scale and a higher density of breeding hens compared to poultry farm X. According to our observations during sampling, raw eggs in farm $\mathrm{C}$ all rolled to the same egg tray and were harvested by hand. Moreover, there was no separate space between the breeding and egg collection areas in farm C. Thus, cross-contaminations may be a major factor responsible for the high prevalence of Salmonella in the eggs collected from this farm. Conversely, in the large-scale and modernized farm X, the production units were completely closed off, with feed supply and egg collection occurring through different conveyor belts; this could reduce the chance of cross-contamination caused by Salmonella present in the environment, especially in the feces of hens. Changes in the management of stocking density at the laying stage can influence Salmonella contamination (Gast et al., 2014). In addition, immunological changes in breeder chickens at the laying stage can increase the contamination rates (Johnston et al., 2012).

Salmonella Typhimurium and S. Enteritidis are the two most commonly identified serotypes and causative agents involved 
in foodborne salmonellosis (Galis et al., 2013; Whiley and Ross, 2015). In the present study, 13 different serotypes were identified from the 61 Salmonella isolates from the eggs. The top four serotypes were $S$. Typhimurium, S. Indiana, $S$. Thompson, and $S$. Enteritidis. Generally, our results were consistent with the results of previous studies reporting that $S$. Typhimurium was the most prevalent serotype in eggs obtained from Penang in Malaysia and 50 poultry farms throughout Korea (Adzitey et al., 2012; Bae et al., 2013). Additionally, $S$. Indiana, $S$. Typhimurium, and $S$. Enteritidis were found to be the predominant Salmonella serovars in eggs sampled in Yangzhou, China ( $\mathrm{Li}$ et al., 2017), while S. Enteritidis was the most prevalent serotype in eggs collected from Shandong, Shanghai, and Guangdong in China (Ni et al., 2018; Xie et al., 2019; Yang et al., 2019). Particularly, S. Enteritidis has always been a common foodborne pathogen associated with salmonellosis caused by consumption of contaminated eggs or egg products, and this is mainly due to its specific survival mechanism in egg white with the assistance of the outer membrane channel TolC (Huang et al., 2019; Raspoet et al., 2019). Here, although S. Enteritidis was not the most dominant serotype in all egg samples, over $70 \%$ of $S$. Enteritidis isolates were derived from poultry farms, and the remaining isolates were recovered from wet markets. It could be considered that $S$. Enteritidis remained to be the predominant serotype of Salmonella in contaminated eggs from poultry farms in the study area.

In total, 10 Salmonella serotypes were detected in the isolates derived from retail eggs, while five serotypes were identified from eggs collected from poultry farms. Both $S$. Typhimurium and $S$. Enteritidis were detected in the eggs from poultry farms and retail markets, whereas the other eight serotypes were detected in the eggs from retail markets only. Interestingly, $S$. Indiana and $S$. Blockley isolates that were commonly detected from poultry farms were absent in the eggs from marketplaces. These results indicate that the prevalent serotypes of Salmonella in eggs differed between retail markets and poultry farms. Previous studies have reported that the pathways of pathogen contamination could be influenced by the egg production, storage, and handling procedures; in other words, cross-contamination may occur during egg storage, transportation, and sales, although some Salmonella strains die in these periods (Namata et al., 2008; Food Drug Administration, 2009; Gast et al., 2014). In addition, S. Indiana accounted for a large proportion $(23.0 \% ; 15 / 61)$ of the Salmonella isolates from eggs in the current study, and it was frequently detected in poultry in China during 2006-2012 (Gong et al., 2017). However, this serotype has not been commonly documented in other countries. Since Salmonella, especially S. Typhimurium, could maintain a high survival rate on eggshells and $S$. Enteritidis within the egg, potential multiple outbreaks associated with these two serotypes in eggs could occur (McAuley et al., 2015). Therefore, apart from improving the hygienic conditions of egg production, efficient measures should be implemented to reduce exposure and surface contact of eggs during transportation and handling in order to prevent contamination by foodborne pathogens including Salmonella.
In poultry rearing, excessive antibiotics are commonly used for disease prevention and growth promotion, leading to the occurrence of antibiotic-resistant bacteria (Krishnasamy et al., 2015; Haskell et al., 2018). A considerable increase in multidrugresistant (MDR) Salmonella has been observed, and the number of MDR Salmonella infections has increased worldwide over the past few years (Fardsanei et al., 2018). In the present study, all the 61 Salmonella isolates from eggs exhibited resistance to one or more antibiotic agents, and only three isolates were nonMDR. Notably, one-third of the isolates were resistant to at least 10 antibiotics. Similarly, Al et al. (2015) found that the rate of MDR Salmonella isolated from eggs and poultry products reached up to $86.5 \%$, and $S$. Thompson isolates exhibited higher antibiotic resistance than isolates of other serotypes recovered from the same marketplace. Moreover, Sanchez-Salazar et al. (2019) obtained 31 Salmonella isolates from layer poultry farms in central Ecuador in 2017, 58.1\% (18/31) of which were MDR. Taken together, these findings corroborate reports showing that MDR Salmonella is prevalent in eggs (Garedew et al., 2015; Bezerra et al., 2016; Taddese et al., 2019). In the current study, almost all $S$. Indiana and $S$. Enteritidis isolates derived from poultry farm $\mathrm{C}$ were MDR strains. This result might be attributed to excessive use and overdosage of antibiotics in poultry farm $\mathrm{C}$ associated with poor production environment and sanitary conditions.

According to the PFGE profiles, some Salmonella isolates within the same serotype were derived from the same location and/or time; these isolates had a close genetic relatedness while they shared the same or similar antibiotic resistance phenotypes. This result is consistent with a previous study of Salmonella contamination in layer poultry farms in Shandong and Hebei Provinces, China, that certain samples within henhouses and eggcollecting areas showed relatively high genetic similarities ( $\mathrm{Li}$ et al., 2018). Here, almost all the Salmonella isolates recovered from poultry farms were identified to be the same serotype with similar PFGE and antibiotic resistance profiles. Likewise, the Salmonella isolated from different backyard eggs in Portugal displayed identical PFGE profiles, indicating that they belonged to a prevalent clone in the region (Ferreiraa et al., 2020). Moreover, some isolates of a certain serotype sampled from different locations and at different times exhibited the same or similar PFGE profiles and antibiotic resistance phenotypes. This result indicates that Salmonella isolates of these serotypes might have existed in the parturient canal of laying hens, poultry rearing environments, or transportation and storage systems for a long period of time. In contrast, some isolates of the same serotype showed diverse PFGE profiles and antibiotic resistance phenotypes. From a poultry slaughterhouse in Brazil, 40 Salmonella isolates obtained over a 20 -week period showed diverse PFGE profiles in the same serotype, except $S$. Enteritidis, which could occur due to the low genetic diversity in this serovar (Dantas et al., 2020). Our results indicate that the prevalence of Salmonella in eggs could pose potential hazards for consumers and result in Salmonella outbreaks over a certain period of time.

This study indicated that Salmonella was prevalent in fresh eggs from poultry farms and retail marketplaces with diverse 
serotypes, and the majority of the isolates were resistant to the multiple antibiotics tested. Some isolates of the same serotype were sampled from the same location and/or time, which shared identical or highly similar PFGE profiles and antibiotic resistance profiles. As eggs play a vital role in daily human life and can be easily contaminated by MDR Salmonella, it is crucial for local health departments to monitor the occurrence of Salmonella in eggs and prevent egg contamination via the food production and supply chains, including poultry farms and retail markets.

\section{DATA AVAILABILITY STATEMENT}

All datasets generated for this study are included in the article/supplementary material.

\section{REFERENCES}

Adzitey, F., Rusul, G., and Huda, N. (2012). Prevalence and antibiotic resistance of Salmonella serovars in ducks, duck rearing and processing environments in Penang, Malaysia. Food Res. Int. 45, 947-952. doi: 10.1016/j.foodres.2011.02. 051

Al, S., Hizlisoy, H., Ertas Onmaz, N., Yildirim, Y., and Gonulalan, Z. (2015). Occurrence and antimicrobial resistance of Salmonella enterica subsp. enterica serovars Typhimurium, Enteritidis, and Typhi isolated from chicken eggs and poultry products. Turk. J. Vet. Anim. Sci. 40, 737-743. doi: 10.3906/vet-1601-17

Andino, A., and Hanning, I. (2015). Salmonella enterica: survival, colonization, and virulence differences among serovars. Sci. World J. 2015:520179. doi: 10.1155/ 2015/520179

Bae, D. H., Dessie, H. K., Baek, H. J., Kim, S. G., Lee, H. S., and Lee, Y. J. (2013). Prevalence and characteristics of Salmonella spp. isolated from poultry slaughterhouses in Korea. J. Vet. Med. Sci. 75, 1193-1200. doi: 10.1292/jvms.130093

Barrow, P. A. (2007). Salmonella infections: immune and non-immune protection with vaccines. Avian Pathol. 36, 1-13. doi: 10.1080/0307945060111 3167

Ben Aissa, R., Al-Gallas, N., Troudi, H., Bethadj, N., and Belhadj, A. (2007). Trends in Salmonella enterica serotypes isolated from human, food, animal, and environment in Tunisia, 1994-2004. J. Infect. 55, 324-339. doi: 10.1016/j.jinf. 2007.06.007

Bezerra, W. G. A., da Silva, I. N. G., Vasconcelos, R. H., Machado, D. N., Lopes, E. D., Lima, S. V. G., et al. (2016). Isolation and antimicrobial resistance of Escherichia coli and Salmonella enterica subsp. enterica in broiler chickens. Acta Sci. Vet. 44, 1-7. doi: 10.22456/1679-9216.80957

Centers for Disease Control and Prevention [CDC] (2013). Standard Operating Procedure for PulseNet PFGE of Escherichia coli O157:H7, Escherichia coli NonO157 (STEC), Salmonella Serotypes, Shigellasonnei and Shigellaflexneri, National Center for Infectious Diseases. Atlanta, GA: Centers for Disease Control and Prevention.

CLSI (2015). Clinical and Laboratory Standards Institute. Performance Standards for Antimicrobial Susceptibility Testing: Twentieth-third Informational Supplement M100-S23. Wayne, PA: Clinical and Laboratory Standards Institute.

Cowen, P., Currier, R. W., and Steele, J. H. (2016). A short history of one health in the United States. Vet. Herit. 39, 1-15.

Cui, M. Q., Xie, M. Y., Qu, Z. N., Zhao, S. J., Wang, J. W., Wang, Y., et al. (2016). Prevalence and antimicrobial resistance of Salmonella isolated from an integrated broiler chicken supply chain in Qingdao China. Food Control 62, 270-276. doi: 10.1016/j.foodcont.2015.10.036

Dantas, S. T. A., Camargo, C. H., Tiba-Casas, M. R., Vivian, R. C., Pinto, J. P. A. N., Pantoja, J. C. F., et al. (2020). Environmental persistence and virulence of Salmonella spp. Isolated from a poultry slaughterhouse. Food Res. Int. 129:108835. doi: 10.1016/j.foodres.2019.108835

\section{AUTHOR CONTRIBUTIONS}

WL conceived the study and drafted the manuscript. WL, HL, $\mathrm{SZ}, \mathrm{ZW}$, and QN performed the experiments and collected the data. HS conducted data analysis. BY supervised the project, experimental design, data collection and analysis, and manuscript preparation. CS and XS guided the experimental design and assisted with manuscript revision. All authors contributed to manuscript revision and agreed to the published version of the manuscript.

\section{FUNDING}

This study was supported by the National Key R\&D Program of China (2017YFC1600100).

FAO (2018a). Available online at: http://faostat3.fao.org/browse/Q/QA/E (accessed June 6, 2018a).

FAO (2018b). Available online at: http://faostat3.fao.org/browse/Q/QL/E (accessed June 6, 2018b).

Fardsanei, F., Dallal, M. M. S., Douraghi, M., Memariani, H., Bakhshi, B., Salehi, T. Z., et al. (2018). Antimicrobial resistance, virulence genes and genetic relatedness of Salmonella enterica serotype Enteritidis isolates recovered from human gastroenteritis in Tehran, Iran. J. Glob. Antimicrob. Resist. 12, 220-226. doi: 10.1016/j.jgar.2017.10.005

Fardsanei, F., Dallal, M. M. S., Douraghi, M., Salehi, T. Z., Mahmoodi, M., Memariani, H., et al. (2017). Genetic diversity and virulence genes of Salmonella enterica subspecies enterica serotype Enteritidis isolated from meats and eggs. Microb. Pathog. 107, 451-456. doi: 10.1016/j.micpath.2017.04.026

FDA (2019a). Investigated Multistate Outbreak of Salmonella Braenderup Linked to Shell Eggs from Rose Acre Farms. Silver Spring, MD: FDA.

FDA (2019b). Investigated Multistate Outbreak of Salmonella Enteritidis Linked to Shell Eggs from Gravel Ridge Farms. Silver Spring, MD: FDA.

FDA (2019c). Investigates Outbreak of Salmonella Oranienburg Linked to Shell Eggs. Silver Spring, MD: FDA.

Fei, X., He, X., Guo, R. X., Yin, C., Geng, H. P., Wu, K. Y., et al. (2017). Analysis of prevalence and CRISPR typing reveals persistent antimicrobialresistant Salmonella infection across chicken breeder farm production stages. Food Control. 77, 102-109. doi: 10.1016/j.foodcont.2017.01.023

Ferreiraa, V., Cardosoa, M. J., Magalhãesa, R., Maiab, R., Neaguc, C., Dumitraşcuc, L., et al. (2020). Occurrence of Salmonella spp. in eggs from backyard chicken flocks in Portugal and Romania - Results of a preliminary study. Food Control. 113:107180. doi: 10.1016/j.foodcont.2020.107180

Food Drug Administration (2009). Prevention of Salmonella enteritidis in shell eggs during production, storage, and transportation. Final rule. Fed. Regist. 74, 33030-33101.

Galis, A. M., Marcq, C., Marlier, D., Portetelle, D., Van, I., Beckers, Y., et al. (2013). Control of Salmonella contamination of shell eggs-Preharvest and postharvest methods: a review. Compr. Rev. Food Sci. Food Saf. 12, 155-182. doi: 10.1111/ 1541-4337.12007

Garedew, L., Hagos, Z., Addis, Z., Tesfaye, R., and Zegeye, B. (2015). Prevalence and antimicrobial susceptibility patterns of Salmonella isolates in association with hygienic status from butcher shops in Gondar town, Ethiopia. Resist. Infect. Control. 4:21. doi: 10.1186/s13756-015-0062-7

Gast, R. K., Guraya, R., Jones, D. R., and Anderson, K. E. (2014). Contamination of eggs by Salmonella enteritidis in experimentally infected laying hens housed in conventional or enriched cages. Poult. Sci. 93, 728-733. doi: 10.3382/ps.201202811

Gong, J., Kelly, P., and Wang, C. (2017). Prevalence and antimicrobial resistance of Salmonella 537 enterica serovar Indiana in China (1984-2016). Zoonoses Public Health 64, 239-251. doi: 10.1111/zph.12328

Hai, D., Yin, X. P., Lu, Z. X., Lv, F. X., Zhao, H. Z., and Bie, X. M. (2020). Occurrence, drug resistance, and virulence genes of Salmonella isolated from 
chicken and eggs. Food Control. 113, 107109. doi: 10.1016/j.foodcont.2020. 107109

Haskell, K. J., Schriever, S. R., Fonoimoana, K. D., Haws, B., Hair, B. B., Wienclaw, T. M., et al. (2018). Antibiotic resistance is lower in Staphylococcus aureus isolated from antibiotic-free raw meat as compared to conventional raw meat. PLoS One 13:e0206712. doi: 10.1371/journal.pone.0206712

Huang, X. Z., Zhou, X. J., Jia, B., Li, N., Jia, J. Y., He, M., et al. (2019). Transcriptional sequencing uncovers survival mechanisms of Salmonella enterica serovar Enteritidis in antibacterial egg white. mSphere 4:e00700-18. doi: $10.1128 / \mathrm{mSphere} .00700-18$

Johnston, C. E., Hartley, C., Salisbury, A. M., and Wigley, P. (2012). Immunological changes at point-of lay increase susceptibility to Salmonella enterica serovar Enteritidis infection in vaccinated chickens. PLoS One 7:e48195. doi: 10.1371/ journal.pone. 0048195

Karimiazar, F., Soltanpour, M. S., Aminzare, M., and Hassanzadazar, H. (2019). Prevalence, genotyping, serotyping, and antibiotic resistance of isolated Salmonella strains from industrial and local eggs in Iran. J. Food Saf. 39:e12585. doi: $10.1111 /$ jfs. 12585

Kasturi, K. N., and Drgon, T. (2017). Real-Time PCR method for detection of Salmonella spp. in environmental samples. Appl. Environ. Microbiol. 83:e0064417. doi: 10.1128/AEM.00644-17

Kingsbury, J. M., Thom, K., Erskine, H., Olsen, L., and Soboleva, T. (2019). Prevalence and genetic analysis of Salmonella enterica from a cross-sectional survey of the new zealand egg production environment. J. Food Prot. 82, 2201-2214. doi: 10.4315/0362-028x.jfp-19-159

Kouam, M. K., Biekop, M. H. F., Katte, B., and Teguia, A. (2018). Salmonella status of table eggs in commercial layer farms in Menoua Division, West region of Cameroon. Food Control. 85, 345-349. doi: 10.1016/j.foodcont.2017.09.037

Krishnasamy, V., Otte, J., and Silbergeld, E. (2015). Antimicrobial use in Chinese swine and broiler poultry production. Antimicrob. Resist. Infect. Control. 4:17. doi: 10.1186/s13756-015-0050-y

Li, Q. C., Yin, K. Q., Xie, X. L., Zhao, F., Xia, J., Chen, Y., et al. (2017). Detection and CRISPR subtyping of Salmonella spp. isolated from whole raw chickens in Yangzhou from China. Food Control. 82, 291-297. doi: 10.1016/j.foodcont.2017. 07.008

Li, R. C., Lai, J., Wang, Y., Liu, S. L., Li, Y., Liu, K. Y., et al. (2013). Prevalence and characterization of Salmonella species isolated from pigs, ducks and chickens in Sichuan Province, China. Int. J. Food Microbiol. 163, 14-18. doi: 10.1016/j. ijfoodmicro.2013.01.020

Li, X. Z., Liu, L., Li, Q. L., Xu, G. Y., and Zheng, J. X. (2018). Salmonella contamination in layer farms in China: detection and genetic analysis. J. Poult. Sci. 55, 1-9. doi: 10.2141/jpsa.0160144

McAuley, C. M., Duffy, L. L., Subasinghe, N., Hogg, G., Coventry, J., and Fegan, N. (2015). Salmonella Typhimurium and Salmonella sofia: growth in and persistence on eggs under production and retail conditions. Biomed. Res. Int. 2015:914987. doi: 10.1155/2015/914987

McWhorter, A. R., and Chousalkar, K. K. (2019). From hatch to egg grading: monitoring of Salmonella shedding in free-range egg production systems. Vet. Res. 50:58. doi: 10.1186/s13567-019-0677-4

Moffatt, C. R. M., Musto, J., and Pingault, N. (2017). Recovery of Salmonella enterica from Australian layer and processing environments following outbreaks linked to eggs. Foodborne Pathog. Dis. 14, 478-482. doi: 10.1089/fpd. 2016.2268

Namata, H., Meroc, E., Aerts, M., Faes, C., Abrahantes, J. C., Imberechts, H., et al. (2008). Salmonella in Belgian laying hens: an identification of risk factors. Prev. Vet. Med. 83, 323-336. doi: 10.1016/j.prevetmed.2007.09.002

Ni, P. E., Xu, Q., Yin, Y. J., Liu, D. L., Zhang, J. M., Wu, Q. P., et al. (2018). Prevalence and characterization of Salmonella serovars isolated from farm products in Shanghai. Food Control. 85, 269-275. doi: 10.1016/j.foodcont.2017. 10.009

Patchanee, P., Eiamsam-ang, T., Vanaseang, J., Boonkhot, P., and Tadee, P. (2017). Determination of regional relationships among Salmonella spp. isolated from retail pork circulating in the Chiang Mai municipality area using a WGS data approach. Int. J. Food Microbiol. 254, 18-24. doi: 10.1016/j.ijfoodmicro.2017.05. 006

Rahn, K., Degrandis, S. A., Clarke, R. C., Mcewen, S. A., Galan, J. E., Ginocchio, C., et al. (1992). Amplification of an invA gene sequence of Salmonellatyphimurium by polymerase chain-reaction as a specific method of detection of Salmonella. Mol. Cell Probes 6, 271-279. doi: 10.1016/0890-8508(92)90002-F

Raspoet, R., Eeckhaut, V., Vermeulen, K., De Smet, L., Wen, Y., Nishino, K., et al. (2019). The Salmonella enteritidis TolC outer membrane channel is essential for egg white survival. Poult. Sci. 98, 2281-2289. doi: 10.3382/ps/pey584

Sanchez-Salazar, E. M., Gudino, M. E., Sevillano, G., Zurita, J., Guerrero-Lopez, R., Jaramillo, K., et al. (2019). Antibiotic resistance of Salmonella strains from layer poultry farms in central Ecuador. J. Appl. Microbiol. 128, 1347-1354. doi: $10.1111 /$ jam. 14562

Taddese, D., Tolosa, T., Deresa, B., Lakow, M., Olani, A., and Shumi, E. (2019). Antibiograms and risk factors of Salmonella isolates from laying hens and eggs in Jimma Town, South Western Ethiopia. BMC Res. Note 12:472. doi: 10.1186/s13104-019-4516-5

U.S. Food and Drug Administration (2013). NARMS Retail Meat Annual Report, 2011. Rockville, MD: U.S. Food and Drug Administration.

Whiley, H., and Ross, K. (2015). Salmonella and eggs: from production to plate. Int. J. Environ. Res. Public. Health. 12, 2543-2556. doi: 10.3390/ijerph120302543

Xie, T. F., Wu, G., He, X. J., Lai, Z. Z., Zhang, H. T., and Zhao, J. (2019). Antimicrobial resistance and genetic diversity of Salmonella enterica from eggs. Food Sci. Nutr. 7, 2847-2853. doi: 10.1002/fsn3.1126

Yang, B. W., Cui, Y., Shi, C., Wang, J. Q., Xia, X. D., Xi, M. L., et al. (2014). Counts, serotypes, and antimicrobial resistance of Salmonella isolates on retail raw poultry in the people's republic of China. J. Food Prot. 77, 894-902. doi: 10.4315/0362-028X.JFP-13-439

Yang, B. W., Qu, D., Zhang, X. L., Shen, J. L., Cui, S. H., Shi, Y., et al. (2010). Prevalence and characterization of Salmonella serovars in retail meats of marketplace in Shaanxi, China. Int. J. Food Microbiol. 141, 63-72. doi: 10.1016/ j.ijfoodmicro.2010.04.015

Yang, J., Ju, Z. J., Yang, Y., Zhao, X. N., Jiang, Z. Y., and Sun, S. H. (2019). Serotype, antimicrobial susceptibility and genotype profiles of Salmonella isolated from duck farms and a slaughterhouse in Shandong province, China. BMC Microbiol. 19:202. doi: 10.1186/s12866-019-1570-Z

Zhang, Q. Q., Ying, G. G., Pan, C. G., Liu, Y. S., and Zhao, J. L. (2015). Comprehensive evaluation of antibiotics emission and fate in the river basins of China: source analysis, multimedia modeling, and linkage to bacterial resistance. Environ. Sci. Technol. 49, 6772-6782. doi: 10.1021/acs.est.5b 00729

Conflict of Interest: The authors declare that the research was conducted in the absence of any commercial or financial relationships that could be construed as a potential conflict of interest.

Copyright (c) 2020 Li, Li, Zheng, Wang, Sheng, Shi, Shi, Niu and Yang. This is an open-access article distributed under the terms of the Creative Commons Attribution License (CC BY). The use, distribution or reproduction in other forums is permitted, provided the original author(s) and the copyright owner(s) are credited and that the original publication in this journal is cited, in accordance with accepted academic practice. No use, distribution or reproduction is permitted which does not comply with these terms. 